\section{Controversial Aspects of the Management of Retinal Detachment}

Edited by C. L. Schepens and C. D. J. Regan. Pp. xxi + 374 illustrated. London: J. \& A. Churchill. 1965. £5 15s.

This book is the outcome of the International conference of the Retina Foundation of Boston, held in May, 1962. The papers and discussions have been edited by Doctors C. L. Schepens and C. D. J. Regan, who state in the preface that they, "have attempted to point out the areas of controversy in the subjects considered and at the same time to minimise the confusion which might puzzle the reader with limited experience in the problems discussed". It is unfortunate that it should have taken three years to present the work of this conference in a form that has lost the impact of the original presentation without gaining the organization of a text-book.

The book is divided into four sections, pre-operative examination, treatment, complications and pathology, and post-operative management and results. Each paper is followed by a discussion section. This arrangement provides some artificial barriers, strikingly obvious in the case of photocoagulation which is the subject of separate papers under the headings of Treatment, and Complications. The subject matter ranges widely, the optics of indirect ophthalmoscopy, the detailed technique of scleral buckling with silicone rubber straps, and the statistics of results are included. Perhaps the most illuminating section is that devoted to the pathology of complications, ischaemic necrosis of the anterior segment is well described and related to interference with the long posterior and anterior ciliary arteries. Glaucoma in relation to detachment surgery, post-operative infection, erosion by implants, and the techniques and complications of the release of subretinal fluid all receive careful consideration.

The book is authoritative; though biased towards American thought it presents international opinion, particularly in the discussions. The information is well documented and deals mostly with established techniques and their hazards. Those practising or training in Ophthalmic surgery will find easily accessible information and opinion to guide them in an enlightened approach to retinal detachment. But the book is of necessity too fragmentary to be of service to inexperienced students of ophthalmology.

\section{Complications after Cataract Surgery}

Edited by Frederick H. THEODORE with 31 contributory authors. Pp. xv +672 , illustrated. London: J. \& A. Churchill. 1965. £5.15s.

This book is a collection of esays by thirty-one contributors, mostly American, under the editorship of Frederick H. Theodore. Each author gives his personal views on a subject of which he has particular experience, chosen within the general framework of the title, "Complications after Cataract Surgery". The style varies from the discursive essay of Arthur Linksz on the "Optical Complications of Aphakia", to the terse, valuable chapter on the "Pathogenesis of Complications following Cataract Surgery" by A. Marvin Gillman, whose tightly packed se trences make heavy reading.

The standard of contributions is high, and the subjects well, though unevenly covered. The nature of the book is such as to commend it strongly to those with a groundwork of knowledge. The chapters on post-operative infection from the diagnostic, therapeutic and prophylactic points of view, contain many new ideas of importance to the practising surgeon. Post-operative mycotic endophthalmitis. culture of anterior chamber aspirates, the place of steroids, and the choice of local and systemic antibiotic combinations are amongst matters discussed.

There is a full exposition of pupillary block in apsakia, with a discussion of its effects on aqueous circulation and the manoeuvres employed to overcome the consequent shallow anterior chamber. Other interesting topics include corneal oedema, post-operative anterior synechia, flat chamber, glaucoma, and medical complications. The operative technique of the division of anterior synechiae and treatment of flat chamber is minutely described, but the technique of cyclodialysis which is a controversial subject, is omitted.

Cataract extraction in the presence of complicating circumstances is considered; these include previous keratoplasty and glaucoma surgery, congenital cataract and lens displacement. Though not strictly within the compass of the title these are interesting contributions. Though not a systematic treatise, the book is of value to the discerning reader, and enlivens the subject with a refreshing diversity of opinion.

\section{Recent Advances in Gastroenterology}

Edited by J. BADENOCH and B. N. BROOKE. Pp. viii + 381, illustrated. London: J. and A. Churchill. 1965. 65s.

During the past twenty years advances in our knowledge of gastroenterology have been so rapid and so extensive that it is difficult even for the practising gastroenterologist to keep pace with all of them.

"Recent Advances in Gastroenterology" fills a gap in the literature on this subject and the Editors are to be congratulated in their choice of subjects and in the manner of their presentation. It is hoped that the book will be re-edited at regular, and not infrequent, intervals.

This work reviews many recent developments in gastroenterology from a clinical aspect, and covers the more scientific aspects of recent advances in language not too technical for the average clinician. It is recommended to practising gastroenterologists, to clinicians practising general medicine and surgery, and to examiners and examinees.

No reference library in a hospital where general medicine and surgery are practised should be without it.

\section{The Year Book of General Surgery (1964-1965 Year Book Series)}

Edited by Michael De Bakey. Pp. 637, illustrated. Chicago: Year Book Medical Publishers. London: Lloyd-Luke. 1965. 64s.

The Year Book of General Surgery 1964-1965 series from Chicago "makes available in detailed abstract form the working essence of the cream of recent international medicoscientific literature". Its distinguished editor is Dr. De Bakey. 120 of the 600 odd pages are devoted to the Heart and Vessels and 200 to the Abdomen. 